\title{
Evaluation of the Sensitivity of Seismic Inversion Algorithms to Different Statistically Estimated Wavelets
}

\author{
V. M. Gomes*, M. A. C. Santos*, R. B. Burgos**, D. M. S. Filho*** \\ *(Department of Geology and Geophysics, Federal Fluminense University, Brazil \\ ** (Department of Structures and Foundations, State University of Rio de Janeiro, Brazil) \\ ***(CENPES/Petrobras, Brazil)
}

\begin{abstract}
Seismic wavelet estimation is an important step in processing and analysis of seismic data. Inversion methods as Narrow-Band and theConstrained Sparse-Spike ones require information about it so that the inversion solution, once it is not a unique problem, may be restricted by comparing the real seismic trace with the synthetic generated by convolution of the estimated reflectivity and wavelet. Besides helping in seismic inversion, a good estimate of the wavelet enables an inverse filter with less uncertainty to be computed in the deconvolution step and while tying well logs, a better correlation between the seismic trace and well log can be achieved. Depending on the use or not of well log information, the methods of wavelet estimation can be divided into two classes: statistical and deterministic. This work aimed to test the sensitivity of acoustic post-stack seismic inversion algorithms to wavelets statistically estimated by two distinct methods.
\end{abstract}

Keywords-Narrow-band, Seismic Inversion, Seismic Wavelet Estimation, CSSI

\section{INTRODUCTION}

Geophysical techniques are used to investigate indirectly the structures and properties associated with the geology of the subsurface. Depending on the features of the desired target, a set of techniques will be chosen. Among the existing methods, the seismic is the most used, considering it provides good resolution even for great depths and allows for estimation of the acousticaland elastic properties of a medium.Estimating these properties involves an approach through Inverse Problem Theory to define a suitable method for the problem at hand. Considering the Earth surface as an acoustic medium a group of methods can be classified as acoustic and post-stack (following classification proposed in [1]). The latter term refers to the fact the data is supposed noiseless and generated through a zero-offset geometry (source and receiver in the same spatial position) a limitation that can be fairly approximated using standard processing flows. The main purpose of these methods is to generate a consistent impedance (i.e. product of density and velocity) model. Under the scope of this work the methods discussed are: Narrow-Band and SparseSpike (CSSI). Narrow-band, occasionally referred as SEISLOG or VERILOG, is the simplest method of seismic inversion being common routine in the 90's [2],[1].CSSI on the other hand, includes more robust methods which allow for a prioriinformation and admits more consistent assumptions about reflectivity. Under a deterministic approach, nowadays even though model-based methods hold as most used, there are still applications of CSSI in light of its robustness as in [3].There is a wide literature on CSSI methods and often they differ only in the deconvolution technique employed to reflectivity estimation. As examples of these, there are the ones that perform deconvolution using maximum likelihood estimation [4],[5],[6]; the L1 norm [7],[8],[9]; and methods developed under a Bayesian point of view [10].The source signature (signal) as a parameter of the seismic experiment is a required input to inversion algorithms. The combination of this signature and the effects on wave propagation is called seismic wavelet. Thus, its estimate from the treated data is a fundamental step to ensure a reliable acoustic model is achieved.

There exists a vast range of works dealing with wavelet estimation techniques and a possible classification is to divide them in deterministic and statistical, their difference being related to the introduction of well $\log$ information. Much of the work in the area until 1996 can be found in [11]. In [11] a solution isproposed using the Kolmogorov method, based on the assumption that the wavelet has minimum phase and its phase spectrum can be obtained by the Hilbert transform of the natural logarithm of the estimated amplitude spectrum. In[13], authorsseek an estimate using the theory of homomorphic systems presented in [14]. The work of[15] seeks a solution to this problem through a strategy based on a simulated annealing algorithm. More recent studies have evolved to more efficient methods at the cost of more complexity as[16], [17] and [18].Sensitivity of the inversion algorithms was analyzed for wavelets estimated using the 
methods: Kolmogorov (or Hilbert Transform)as in [12] and a combination of smoothing the seismogram's amplitude spectrum and setting a constant linear model to the phase spectrum, similar to the methodology used in [19]. To reduce uncertainty in the phase estimate, after estimation a correction in the phase spectrum based on[20] was applied. In this work it was possible to analyze the behavior of the seismic inversion methods: Narrowband and Bayesian, regarding wavelets estimated by the aforementioned methods and noise. In general the second got far superior results, admitting more robust estimates of the impedance in all tests. For wavelet estimation methods, the second (smoothing) obtained better results, although for both, the phase estimates were not good.

\section{THEORETICAL BACKGROUND}

\section{A. Convolutional Model}

The convolutional model in its simplest form was originally described in [12] and is a valid approximation for several steps of seismic processing. Its validity depends on some assumptions two of which are: (1) the reflectivity is a random process and (2) the wavelet has minimum phase. Considering a reflectivity series $r$ and a wavelet $w$, the seismic trace or seismogram $s$ can be mathematically represented as:

$$
\begin{aligned}
& s_{t}=\sum_{k=0}^{\infty} w_{k} r_{t-k} \text { or } \quad s(t) \\
&=w(t) * r(t)
\end{aligned}
$$

It can be viewed as an approximation to an ideal zero-offset acquisition where the wavelet is an idealization of a waveform that could account both for the one created by the source and all the wavefield propagation phenomena changing it along time (i.e. dispersive effects). As long as a suitable processing workflow is applied to the recorded data, the final post-stack section or volume reasonably satisfies the convolutional model assumptions thus admitting inversion with the latter discussed methods.

\section{B. Wavelet estimation}

The two methods discussed here are: Hilbert transform method and Smooth spectra method. Before going further the fact they are a good approximation to wavelet amplitude spectrum while producing a poor estimate of phase spectra must be emphasized. To overcome this drawback of both methods a correction based on the Automatic Phase Correction (APC as referred herein) introduced in [20] was adopted. In the original APC the wavelet phase spectrum is rotated by a constant angle and a synthetic calculated for each rotation until an entropy norm is maximized. Here the best solution was found considering the minimum error norm seeking an increase in correlation.

\section{1) The Hilbert Transformor Kolmogorov factorization method}

This method estimates the wavelet amplitude spectrum of its minimum phase version considering the data auto-correlation. However the actual phase might not be minimum thus phase spectrum must also be estimated. Using the convolutional model and assuming reflectivity as a random (white Gaussian) series, the wavelet autocorrelation can be fairly approximated by the seismogram's one, differing only by a scale factor equal to the total energy of reflectivity, as shown in [21].

As stated in [22], calculation of spectral density of a signal can be accomplished by the Fourier transform (FT) of its auto-correlation $(\phi)$ and by the square of its magnitude spectrum $(|W(\omega)|)$ which are related through:

$|W(\omega)|=\sqrt{|\phi(\omega)|}$

Therefore using the above relation an estimate of a minimum phase wavelet amplitude spectrum can be found from the square root of the seismogram's spectral density.

The phase spectrum is obtained using the equation derived in [12]:

$\Varangle W(\omega)=$

$-2 \sum_{1}^{\infty}[\sin (\omega t)]\left[\frac{1}{2 \pi} \int_{0}^{\pi} \cos (\omega t) \ln |W(\omega)| d \omega\right]$

Derivation of the above equation can be done using the Hilbert transform of the logarithm of the wavelet's amplitude spectrum as stated in [23] thus the method's name.

\section{2) Smoothing Spectra method}

The theory behind this method is simpler than the previous one. Under the assumption reflectivity is a white Gaussian series and the wavelet has a band-limited smooth varying amplitude spectrum, the smooth "trend" of the seismogram's amplitude spectrum would be associated with the wavelet. Though simple it holds for most of the cases hence leading to good wavelet estimates. Numerical implementation involves the choice of a smoothing operator to the amplitude spectrum of the seismogram and a minimum, mixed or maximum phase guess for the spectra, where the most common is the selection of a constant value. The parameter that most influences the final result is the operator's size, which can be a simple moving average filter.

\section{Seismic inversion methods}

For seismic data inversion Narrow-band and a Bayesian method were used, both discussed below. 


\section{1) Narrow-band inversion}

Considering a wavelet, an estimated earth reflectivity and an initial value for most superficial layer a recursive equation can be used to calculate an impedance model iteratively using the index $i$ :

$r_{i}=\frac{Z_{i+1}-Z_{i}}{Z_{i+1}+Z_{i}}$

From algebraic manipulation of (4) we achieve:

$Z_{i+1}=Z_{i} \frac{1+r_{i}}{1-r_{i}}$

(5)represents a recursive process for obtaining the acoustic impedances of subsurface and is the base for several seismic inversion methods.

The big difference between the Narrowband method other recursive involves the choice ofdeconvolution technique used for reflectivity estimation. In this specific case, techniques following the theory of optimum Wiener filters[24], are adopted. The deconvolution filers are constructed in an adaptive way, being however highly sensitive to noise. Seismic deconvolution comes from image processing theory, citing Yilmaz[21], "it is responsible for estimating the reflectivity by compressing the wavelet and attenuating reverberations and short period multiples, hence providing greater temporal resolution".Optimum Wiener deconvolution in the broad sense of Narrow-band inversion has a very low computational cost. However for seismic inversion purposes it represents the worst alternative due to the limited bandwidth property, characteristic of the estimated reflectivity. Low frequency loss represents absence of information regarding the main trend of the geological model of subsurface, while the loss of high frequency components leads to reduction in temporal resolution. This problem is extensively discussed in literature, e.g.[1], and as it is not the focus of this paper, a workaround would be to use a wavelet with small central frequency to ensure the low frequency part of the spectrum is present.

\section{2) A Bayesian approach (CSSI)}

Seeking to overcome the limited bandwidth problem discussed above, the sparse-spike methods emerged. Considering the deconvolution algorithms used, they will try to estimate a reflectivity comprised of sparse deltas, ensuring an increase in the estimated frequency band [10]. Sparsity will be provided by constraining the estimated model through minimization of a some sparse norm (e.g. L1, Huber,Cauchy). Considering the inversion methods, a bigger band will lead to less uncertainty in the results. Additionally toincreasing the bandwidth through the sparsity assumption,a set of constraints are usually input as a priori information, commonly low frequency information (a smooth model) calculated from well logs. Nevertheless improvement is associated with higher complexity algorithms and consequently a rise in computational cost, which in this case become irrelevant compared to the robustness of results. In a Bayesian framework both sparsity regularization norms and constraint dependence can be introduced in the objective function describing the problem. As in [10] a blocky impedance model can be found from minimization of the functional:

$$
\begin{aligned}
J=\kappa J_{c} \frac{1}{2} \| \frac{1}{\sigma}(W r & -s) \|^{2} \\
& +\frac{1}{2}\left\|N^{-1}(C r-\mathrm{B})\right\|^{2}
\end{aligned}
$$

In $(6) \kappa$ is a hyperparameter that ponders sparsity in reflectivity, $J_{c}$ is the sparsity norm, $\sigma$ is an estimate of the noise level, $W$ is the convolution matrix associated with the wavelet, $r$ is the reflectivity, $s$ the observed seismic data, $C$ is an integration operator and $\mathrm{B}$ is the natural logarithm of the normalized impedance or double the cumulative sum of reflectivity. The term $N$ is the diagonal matrix $N_{k, k}=\vartheta_{k}$ where $\vartheta_{k}$ can be seen as a vector of the uncertainties associated with a priori information, thus $N$ imposes some restrictions on the estimated model.

Following the options for the sparsity norm in [10] the Cauchy norm was chosen,

$J_{\text {Cauc hy }}=\frac{1}{2} \sum_{k} \ln \left(1+\frac{r_{k}{ }^{2}}{\vartheta_{k}^{2}}\right)$

Finally, it is noteworthy to comment that the choice of parameters $\kappa$ and $\sigma$ as well as the initial model must be carefully made to ensure convergence to the solution or to the global minimum of the objective function.

\section{NUMERICAL EXPERIMENTS}

The methodology used for sensitivity testing, in general, involved modeling of a synthetic seismogram from an input model, wavelet estimationand finally the inversion.The acoustic model assumed here is the Marmousi, shown in Fig.1. It was created in 1988 by the French Petroleum Institute, based on a geological profile in the Kwanza basin in North Quenguela. It represents a complex geological environment and is one of the most widespread models for seismic analysis, modelling and inversion, hence its choice.For modelling of the reference (observed) seismogram the convolution of reflectivity with a Ricker wavelet with center frequency of $10 \mathrm{~Hz}$ was used. The choice of this value is a way to overcome the problem of absence of low frequencies as previously discussed. The results can be divided into two stages; the first was analysis of the wavelet estimation methods the second of inversion techniques. Results of the wavelet estimation considered synthetic models generated with minimum and zero phase wavelets 
(Fig.2), both tests admittingalso the existence of noise. The inversion analysis used a seismogram modeled with a zero phase wavelets as reference and also accounted for noise influence. The noise used in both analyses is white Gaussianwith standard deviation $\sigma=10^{-3}$.

\section{Wavelet estimation analysis}

As stated above, the tests to estimate the wavelet were performed assuming data modeled with minimum and zero phase Ricker wavelets. Fig.2show modeled traces without noise. For the Hilbert transform method, the results are shown in figures Fig.3andFig.4. As expected, better results are reached for the minimum phase situation. For the zero phase case even after APC the estimated wavelet does not have a shape similar to the true. It was discussed in the subsection B that this method depends on the assumption that the wavelet is minimum phase, which explains the results. Moreover noise addition to the trace worsens the algorithm's performance, creating artifacts on the shape of the estimated wavelet and for the zero phase case satisfactory results cannot be achieved showing the method is highly sensitive to noise.
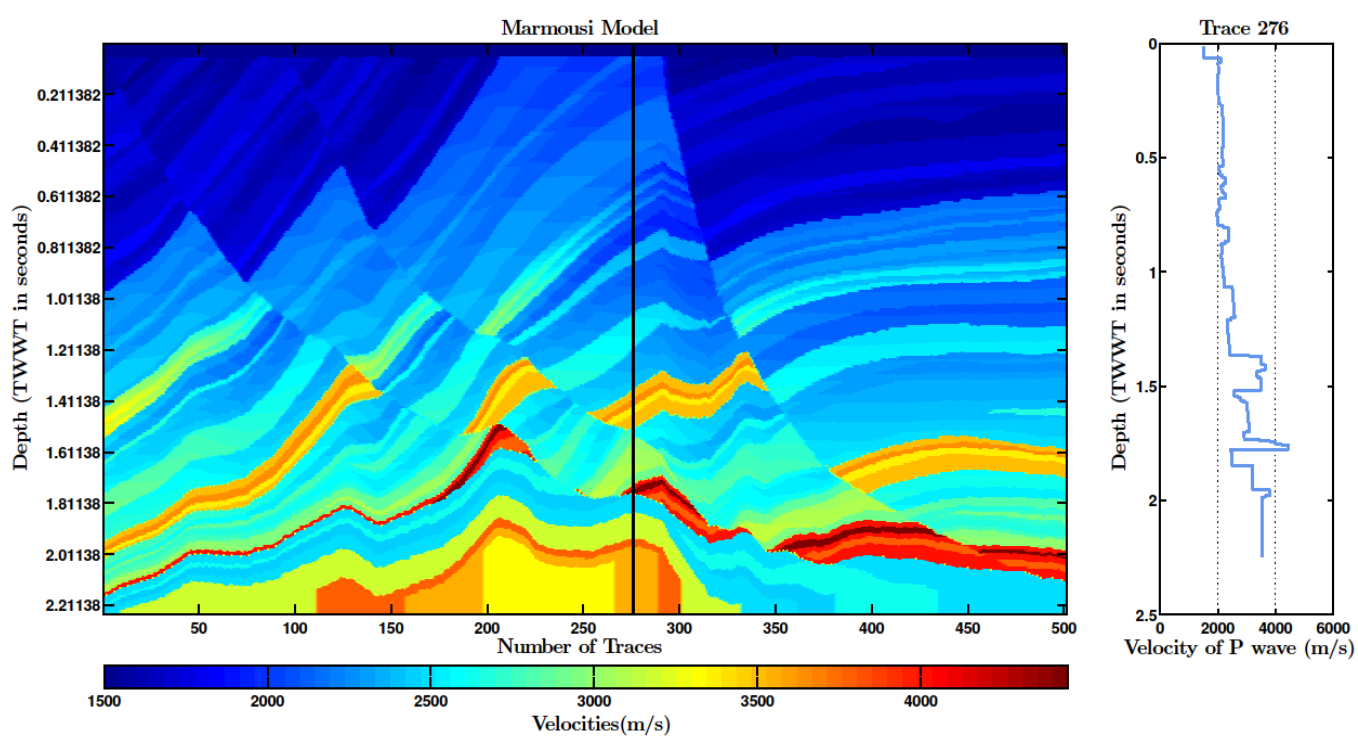

Fig.1 -Marmousi model and trace 276 in two-way-travel-time.
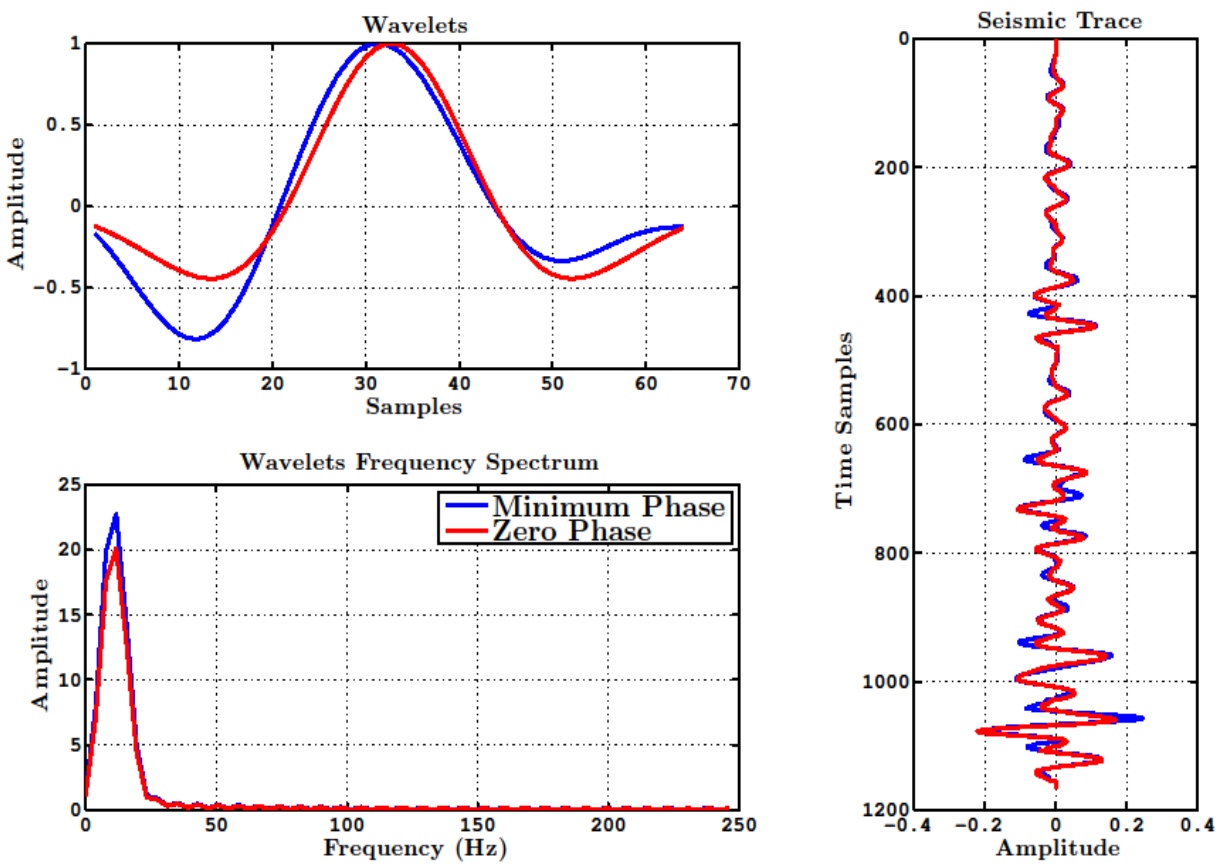

Fig.2 - Ricker wavelets with zero and minimum phase, their respective amplitude spectrum and modeled traces 

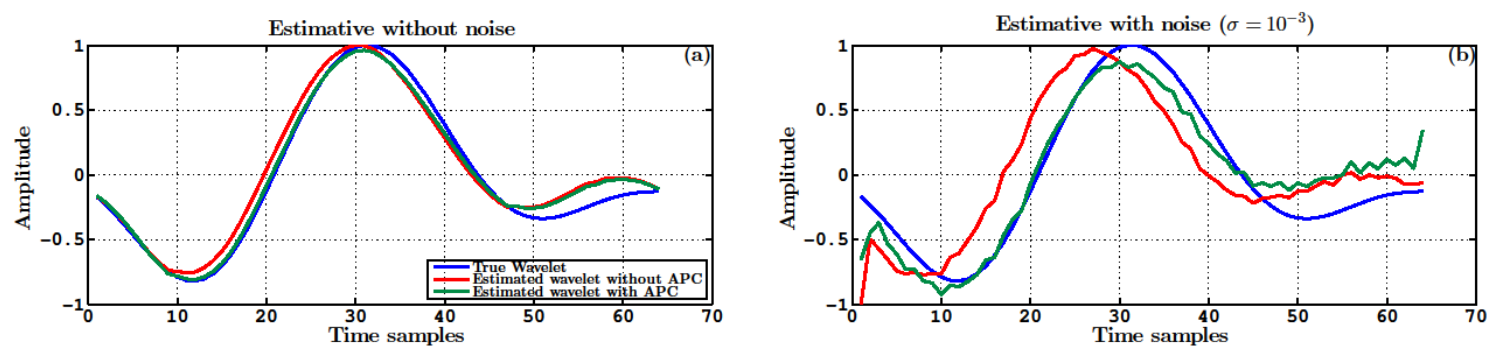

Fig.3 -Minimum phase ricker wavelet estimation with Hilbert transform method. (a) without and (b) with noise
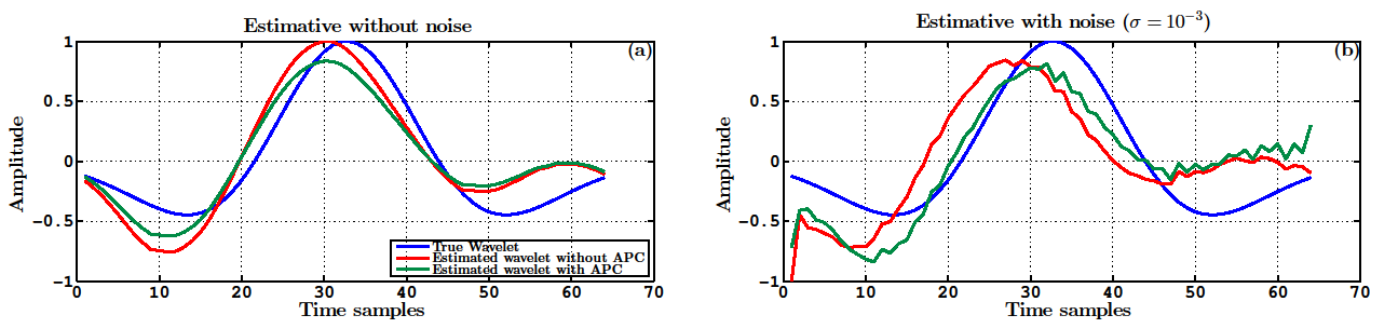

Fig.4 -Zero phase ricker wavelet estimation with Hilbert transform methods. (a) without and (b) with noise.

Analyzing the results of estimation with the Smoothing spectra method, shown in Fig.5 and Fig.6, it can be noted that the presence of noise doesn't have as much influence on the results as for the previous method. Also, as there is not a dependency of results with assumptions about the phase, the APC correction proved more effective for this technique. However, it can be seen that the best estimates for the shape and phase spectrum correspond to the zero phase case.It was observed that better performance of the method is achieved when guessing a linear phase spectrum for the estimated wavelet before APC. The best results for the zero phase case were obtained when considering small values inside the interval [1,2] for the phase spectrum; for the minimum phase case, larger values achieved more success. Adopting a moving average filter for spectral smoothing is largely responsible for attenuation of noise effects as the shape artifacts as seen for the previous method.
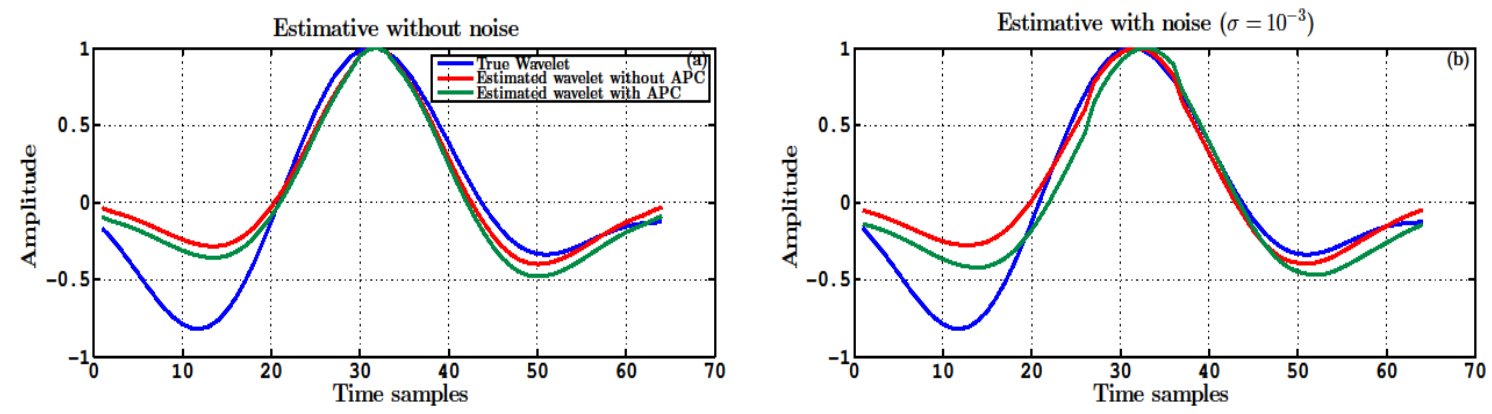

Fig.5 -Minimum phase ricker wavelet estimation using Smooth spectra method. (a) without and (b) with noise.
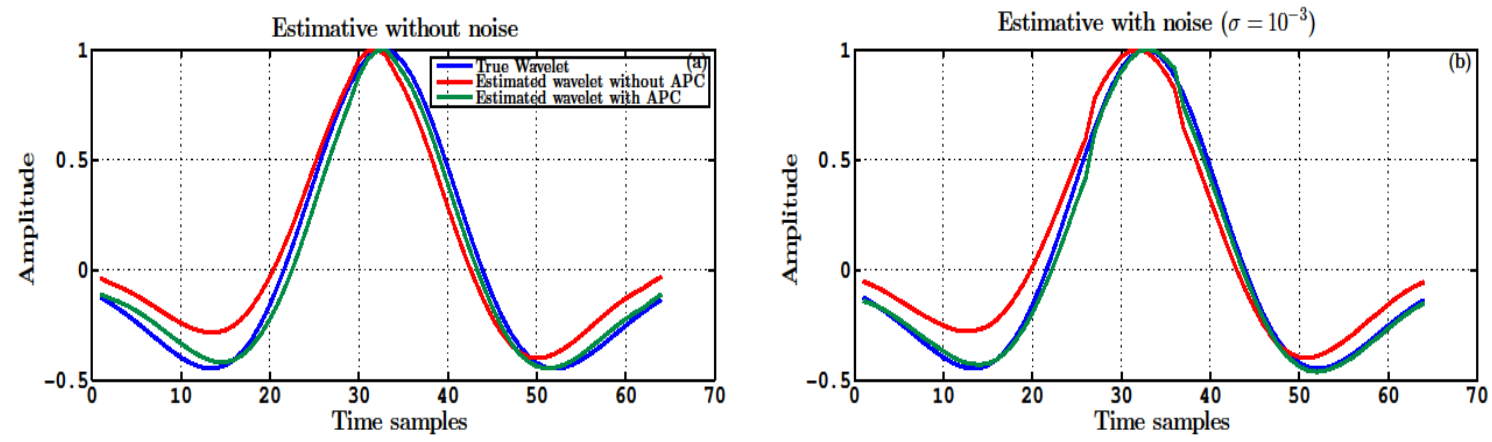

Fig.6 -Zero phase ricker wavelet estimation using Smooth spectra method. (a) without and (b) with noise. 
In general the second method can achieve an estimate with less uncertainty for the wavelet when analyzed with respect to noise and phase. An option for improvement of the results of both techniques would be to use a more suitable algorithm for correction and estimation of the phase spectrum considering the estimated phase spectra are far from the real (Fig.7,Fig.8,Fig.9 andFig.10) while good estimates were obtained for the amplitude spectrum, as shown by Fig.11, Fig.12, Fig.13 and Fig.14.
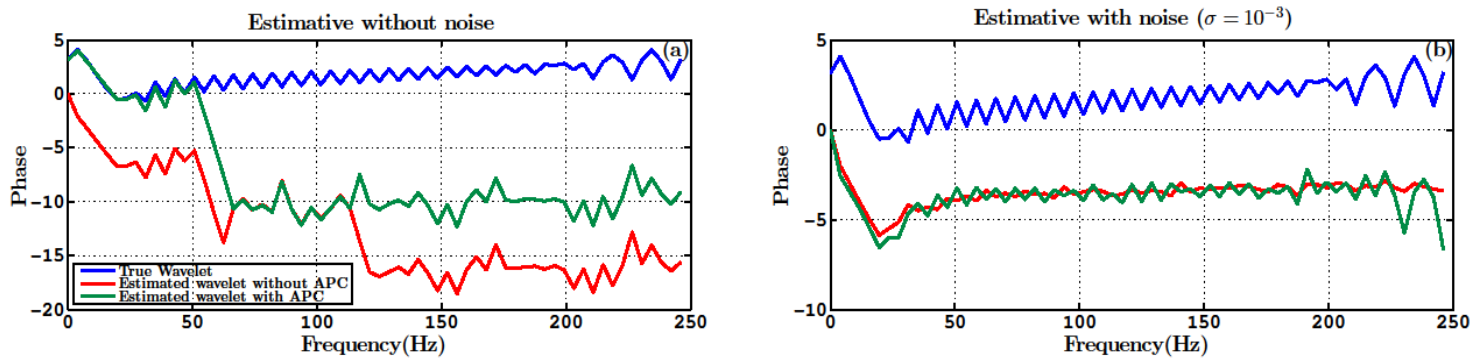

Fig.7 -Phase spectra of minimum phase ricker wavelet estimated with Hilbert transform method. (a) without and (b) with noise.
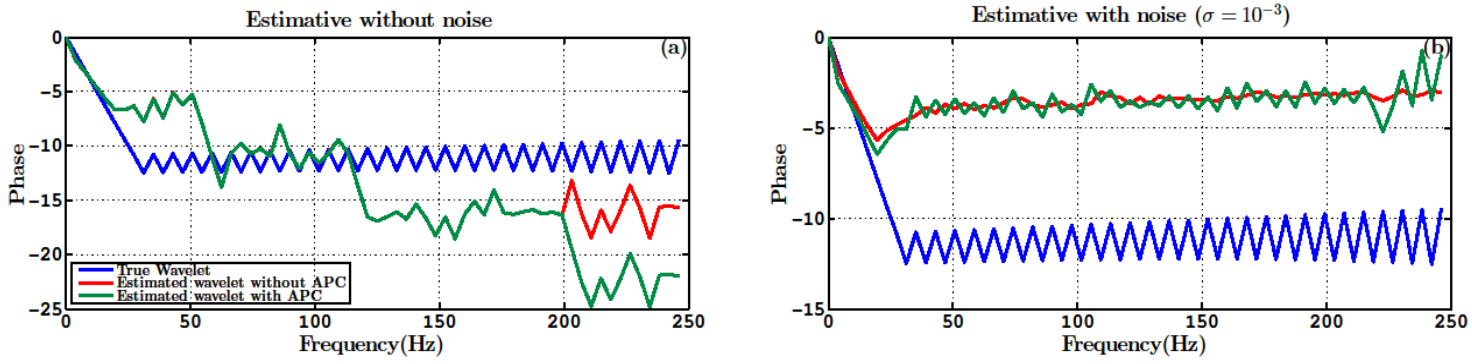

Fig.8 -Phase spectra of zero phase ricker wavelet estimated with Hilbert transform method. (a) without and (b) with noise.
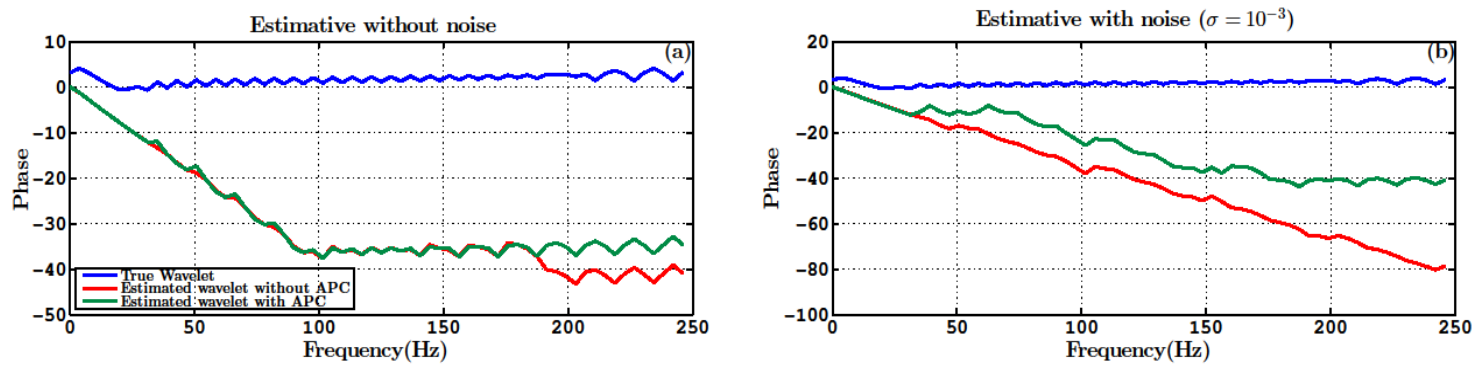

Fig.9 -Phase spectra of minimum phase ricker wavelet estimated with Smooth spectra method. (a) without and (b) with noise.
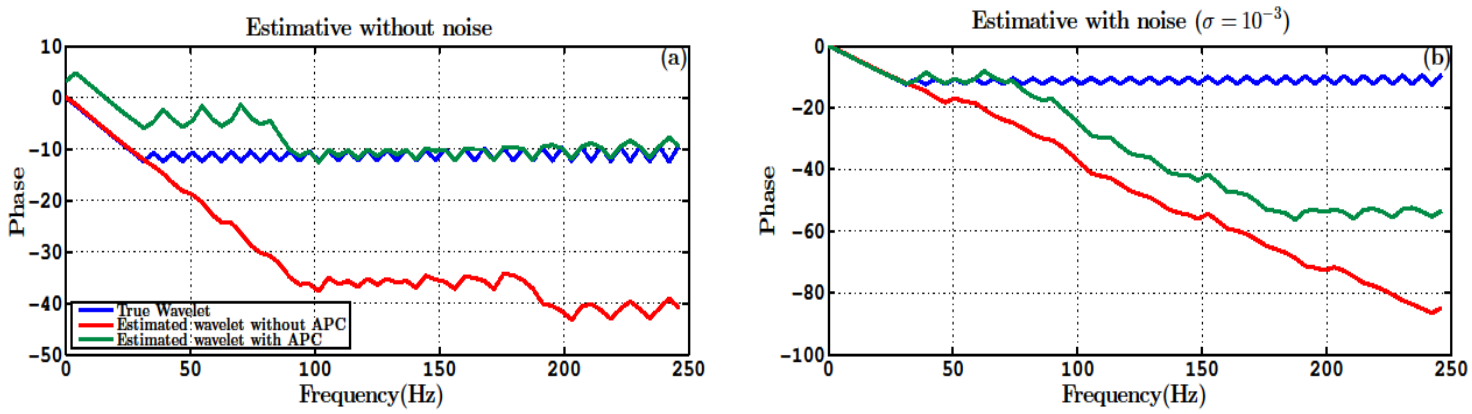

Fig.10 -Phase spectra of zero phase ricker wavelet estimated with Smooth spectra method. (a) without and (b) with noise. 

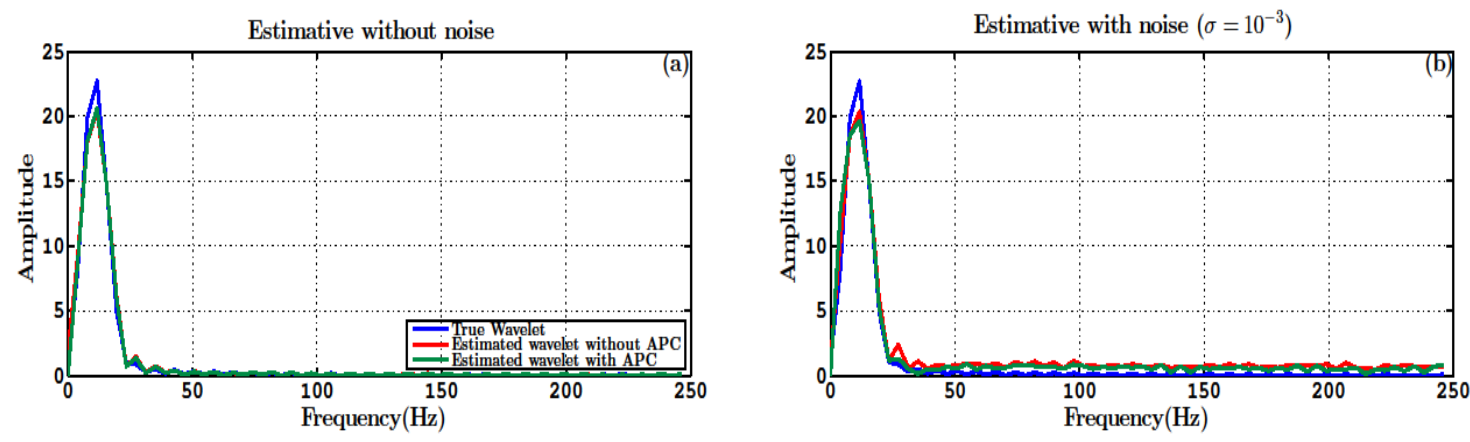

Fig.11 -Amplitude spectra of minimum phase ricker wavelet estimated with Hilbert transform method. (a) without and (b) with noise.
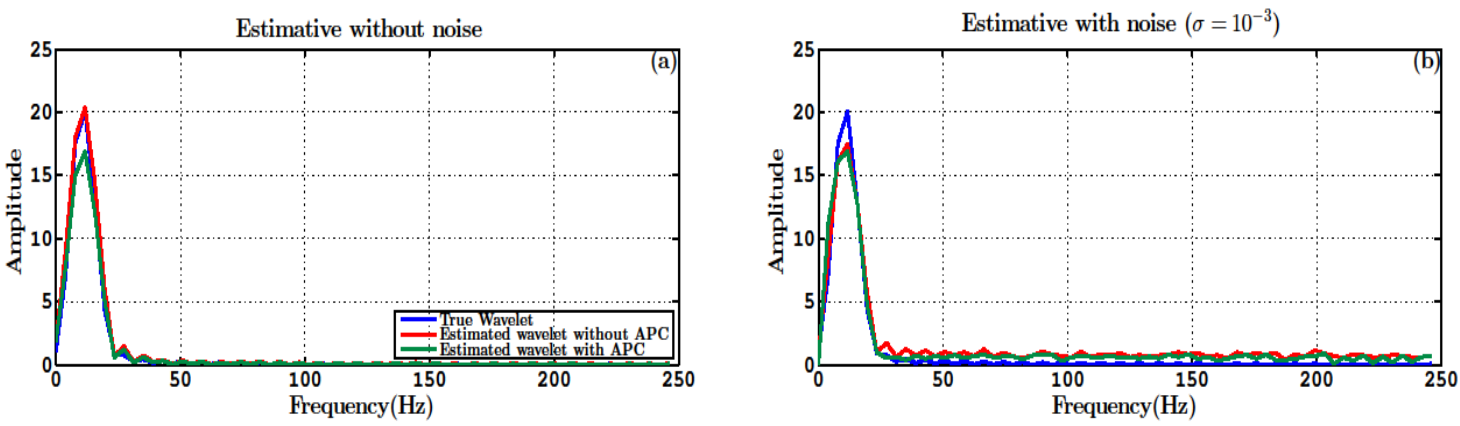

Fig.12 -Amplitude spectra of zero phase ricker wavelet estimated with Hilbert transform method. (a) without and (b) with noise.
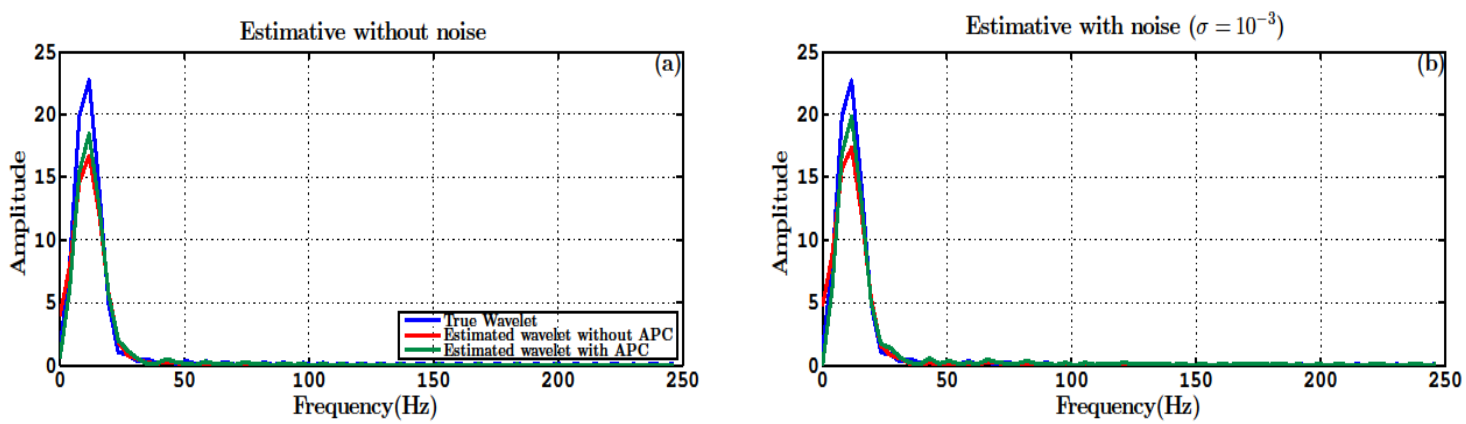

Fig.13 -Amplitude spectra of minimum phase ricker wavelet estimated with Smooth spectra method. (a) without and (b) with noise.
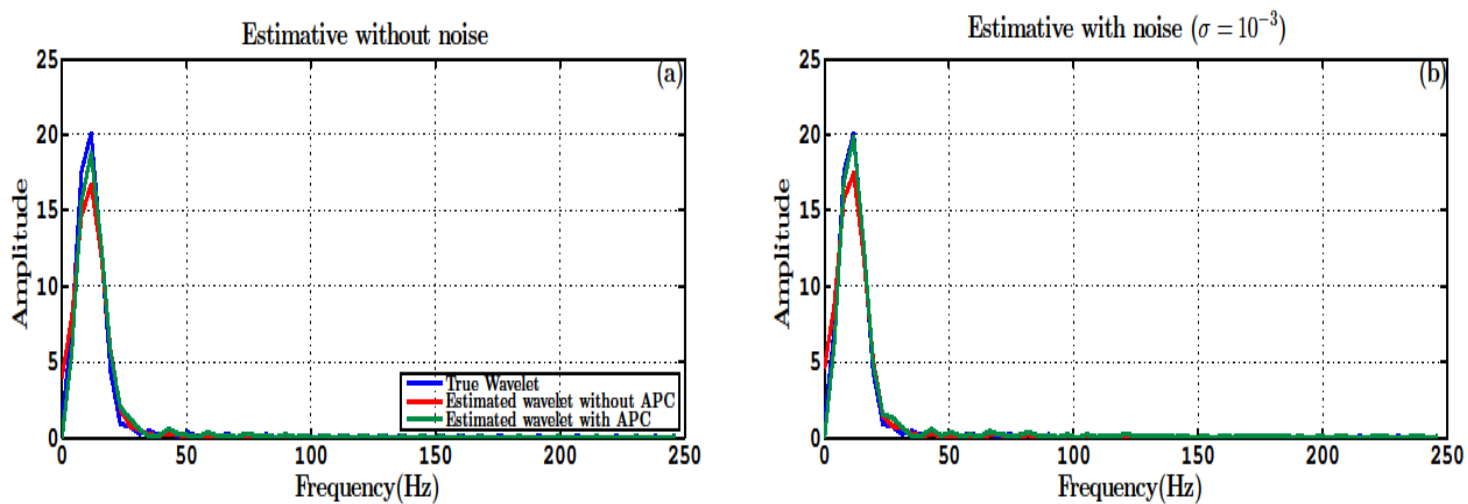

Fig.14 -Amplitude spectra of zero phase ricker wavelet estimated with Smooth spectra method. (a) without and (b) with noise. 


\section{E. Seismic inversion analysis}

As previously stated synthetics used as the observed (reference) data for the inversion analysis were modeled with a zero phase wavelet. For simplicity, density was assumed to be constant so that the estimates, even when referred to as impedance, are the velocities of $\mathrm{P}$-wave propagation. Results are presented and discussed below. First the performance of the methods for situations when noise is present is analyzed, then their sensitivity to the estimated wavelets is observed. Considering the Narrow-band method for the case where the real wavelet is used, Fig. 15 and 16show that problem is solved even in the presence of noise, although the results are not as good as in the absence of it. It can be concluded that if the wavelet is well estimated, even in the presence of noise good results are obtained. In

Fig.16 this idea is well represented, showing the similarity between the estimated and synthetic models. It is necessary to discuss here the parameter $\mu$ which represents knowledge about the noise. A good estimate will lead to results with less uncertainty. One can think of it as a regularization parameter to the inversion since the results are highly influenced by its choice. This constant will also act on situations with estimated wavelets, even in the absence of noise, as will be seen ahead, once these wavelets carry an amount of uncertainty associated with the estimation process which will be interpreted as noise by the inversion algorithm. It was observed that for the tests with noise addition $\sigma=10^{-3}$, a value that achieve good results is $\mu=0.1$
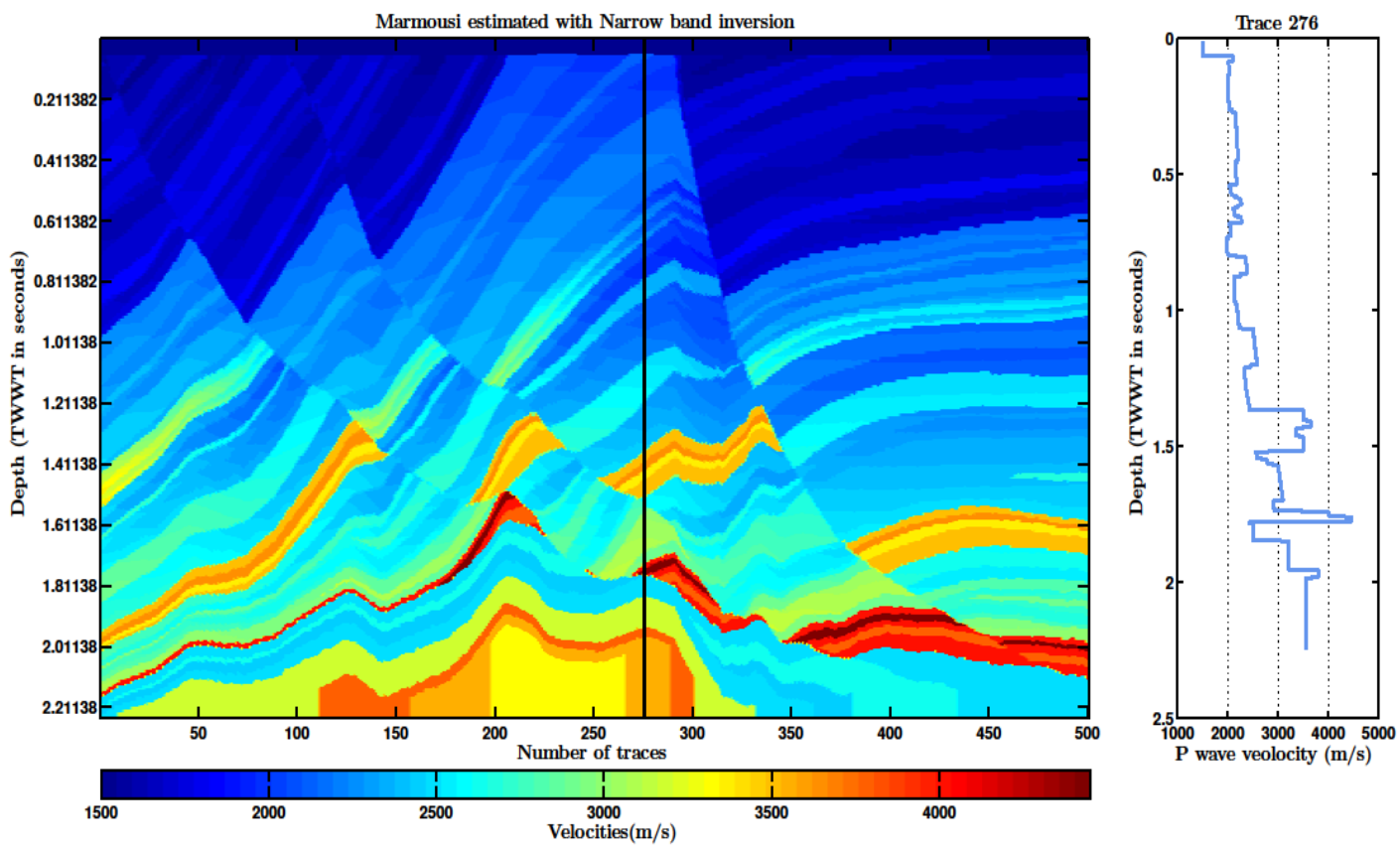

Fig.15 -Narrow-band inversion with true wavelet and without noise addition.

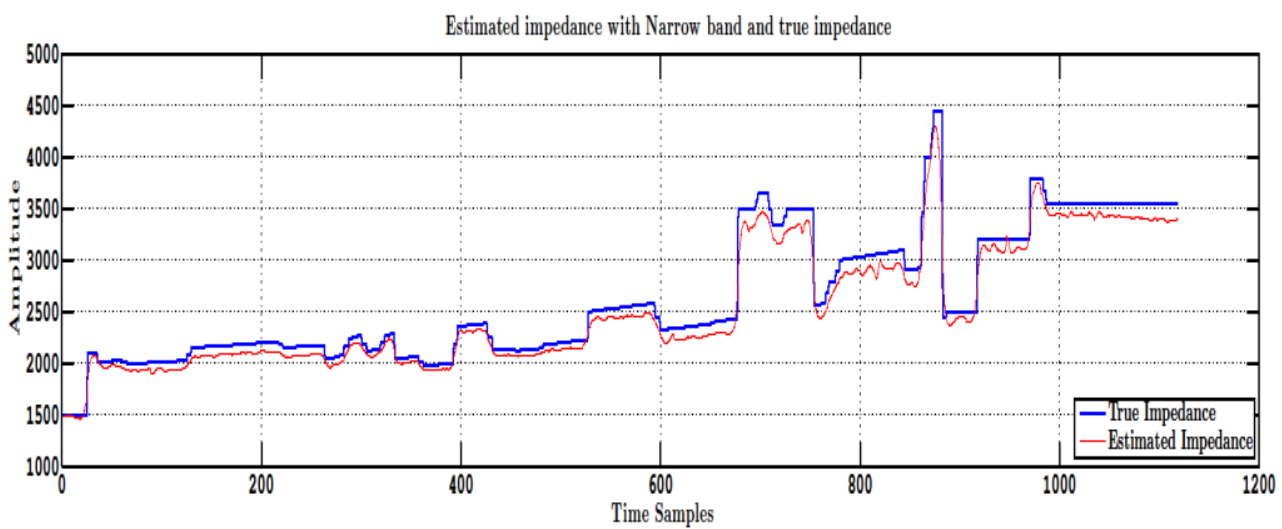

Fig.16 -Narrow-band inversion with true wavelet and with noise $\left(\sigma=10^{-3}\right)$ addition for trace 276. 
The use of an estimated wavelet, contrary to the above results, will not produce such reliable results as previously discussed. Figs. 17, 18, 19 and 20 show the results to the sensitivity of the Narrowband inversion to the estimated wavelets. The striking effect in all cases is the discrepancy between amplitudes, although interfaces have been well delimitated. This effect on the other hand is related to the previously mentioned problem of lack of low frequencies, in fact, during the tests it was observed that lowering the central frequency of the wavelet to5Hz produces better results. It must be noted that the method has not failed; it succeeded in identifying the most significant subsurface interfaces.Nevertheless since this method does not use an initial model this behavior is something one should expect in absence of low frequencies, therefore a solution would be to add the low frequency impedance model to the final estimate from Narrow-band inversion. For results with noise and an estimated wavelet, the noise does not show significant influence when a good estimate for $\mu$ was used. This confirms the earlier statement that the method can solve, to some extent, effects related to the presence of noise. It could also be noticed that, the results using a wavelet estimated by the Hilbert method are more sensitive to noise, requiring larger values of $\mu$ in comparison with the Smooth spectra ones..

Comparison of the results using different estimation techniques again shows that the results of Smooth are superior (

Fig.17 and
Fig.19). As a final statement regarding Narrow-band inversion results, it must be pointed out the need to estimate a value for $\mu$ even in the absence of noise. This relates to numerical noise introduced by the use of the Discrete Fourier Transform algorithm (calculation of the deconvolution step is performed in the frequency domain). Looking now at the results of the Bayesian method, Fig.21, Fig.22 and Fig.22Fig. 23 show the inversion results using the true wavelet. One can see that the method can obtain good estimates for impedance even if noise is present. In general, the algorithm converged well to all methods.The first discussion regarding the Bayesian inversion must be about the parameters chosen for the purpose of ensuring better results. In (6) and (7) all parameters involved are shown, however the ones actually used as input to the algorithm are: an estimate of the standard noise deviation $(\sigma)$ and the uncertainty vector associated with the a priori information $(\vartheta)$. Nonetheless, for each test performed a new set of parameters should be selected once each setting possesses different levels of uncertainty. Complementing the discussion in the paragraph above, it is necessary to talk about the use of an initial impedance model. The one used here was generated by smoothing the true model. It is responsible to introduce a priori information and consequently increase bandwidth, therefore ensuring better convergence. As previously discussed, the low frequency components carry relevant information about trends of subsurface geology and thereby, use of an initial model allowed incorporation of these components and hence of the actual impedance trend, constraining the results as observed in the tests.

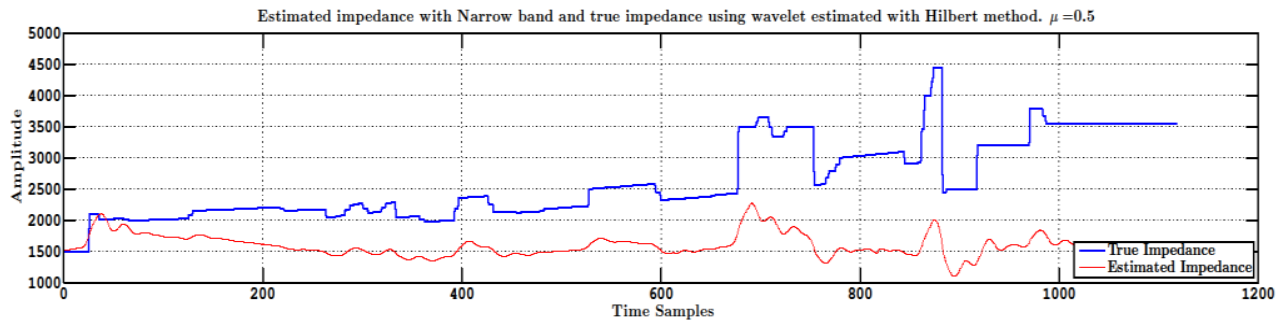

Fig.17 - Narrow-band inversion with wavelet estimated using Hilbert transform method for trace 276.

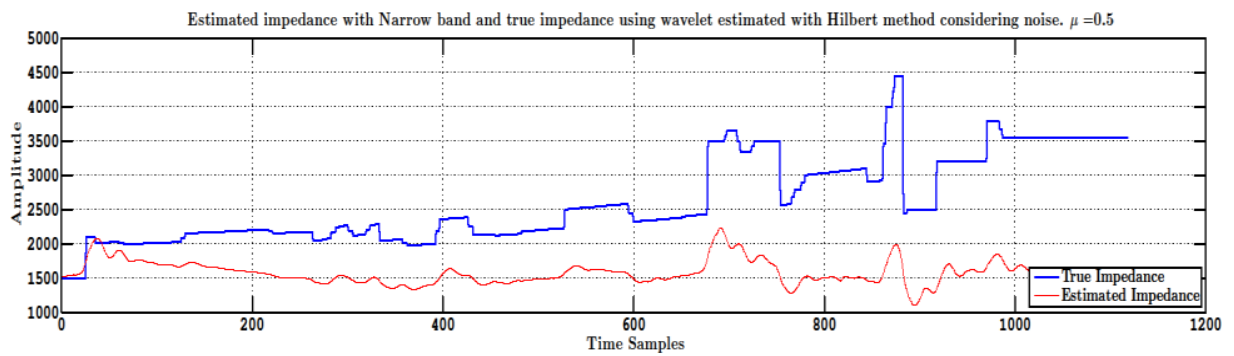


Fig.18 -Narrow-band inversion with wavelet estimated using Hilbert transform method and noise $\left(\boldsymbol{\sigma}=\mathbf{1 0}^{-\mathbf{3}}\right)$ addition for trace 276

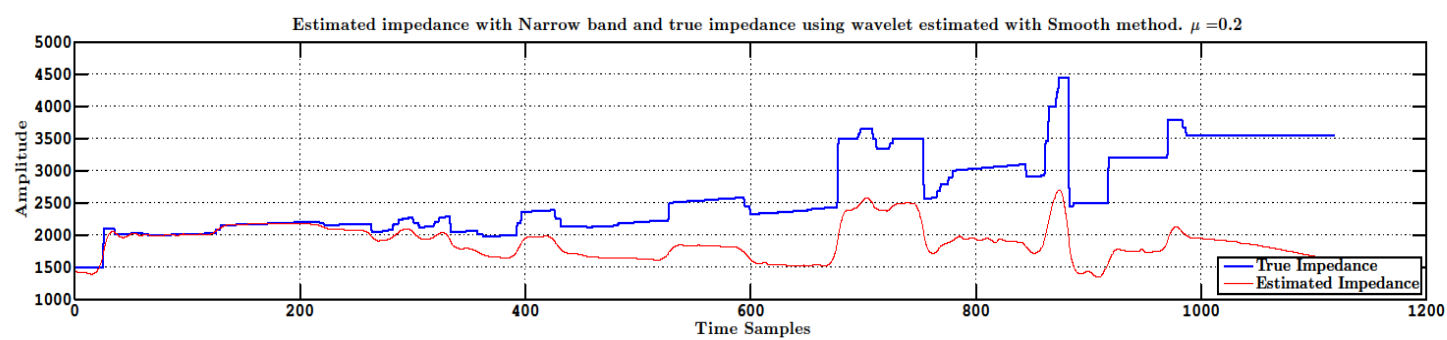

Fig.19 -Narrow-band inversion with wavelet estimated using Smooth spectra method for trace 276.

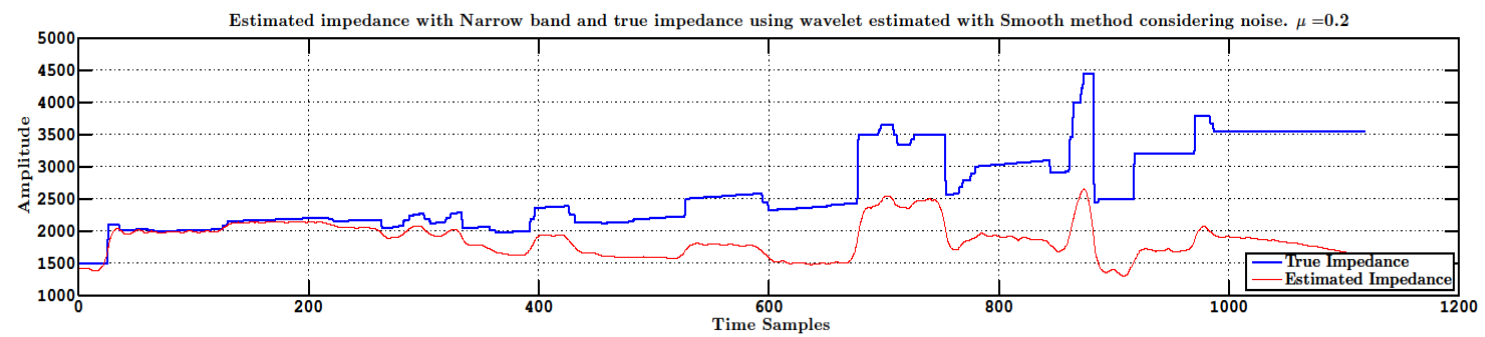

Fig.20 -Narrow-band inversion with wavelet estimated using Smooth spectra method and noise $\left(\sigma=10^{-3}\right)$ addition for trace 276

By observing the influence of noise, Fig.23, Fig.24 and Fig.25 prove the method as more efficient than the previous to deal with these situations, even when using estimated wavelets. A characteristic effect of noise are the artifacts with oscillatory behavior in the calculated impedance, easily noticeable in the last two above-mentioned figures what however, does not prevent the identification of the main interfaces. Finally it is reiterated that the good performance of the method is highly dependent on the above mentioned parameters so that, good results can only be achieved through a good estimate of these.Regarding the performance tests with estimated wavelets, again this method outperforms the Narrow Band, achieving good results for the two estimation techniques. In tests with the noise, however, the presence of the artifacts discussed is significant, especially when using wavelets estimated by Hilbert transform method (Fig.24). By observing the results, once again the Smooth appeared as the best alternative, although in the tests discrepancies were not as evident, showing that the Bayesian can solve for uncertainties associated with the estimation of the wavelet. Fig.26 and Fig.27 illustrate the differences for results with both estimated wavelets.

As a final discussion about this method, the artifacts in Fig. Fig.21 will be treated. They emerge as a consequence of numerical instability during the Conjugate Gradient optimization. As the inversion is performed trace by trace, situations where the method cannot converge well or when errors associated with numerical approximation appear might happen, justifying the artifacts in a few traces. Nonetheless, the Conjugate Gradient method minimizes the misfit with a step size per iteration, the search for this step size is referred as line search and was implemented here in a somehow empirical way, where from an initial value the next was found through fitting a polynomial between the previous and values closer to it. Therefore the selected step size could go over the global minimum hence increasing the error and leading to the discussed discontinuities.Comparison of the above results show that Bayesian inversion has guaranteed less estimation errors, proving superior to Narrow-band for all tests. However, it is necessary to mention that its computational cost is higher, in some cases requiring more than 100 iterations for convergence to an acceptable result. In any case, its sensitivity to the uncertainties introduced by noise and estimated wavelet is smaller, what can justify its choice over the Narrow-band.

\section{CONCLUSION}

Arising from the need to obtain estimates of the parameters associated with subsurface geology mainly with the purpose of exploration, geophysical methods have increasingly gained space once it is an indirect way and therefore more economical. In the case of the seismic method, probably the most popular, information on acoustic properties can be obtained using seismic inversion algorithms, among which two were discussed here, Narrow band and Bayesian.The proposed methodology aimed to test the sensitivity of the inversion methods considering 
estimated wavelets and noise. Comparison of the wavelet estimation methods showed that Smooth produces better results. However, the amplitude spectrum of the estimated wavelets showed that both can solve the problem. Phase spectrum analysis indicates otherwise, so that even after APC phase correction the spectrum was not well approximated. Techniques that are able to produce better estimates to the phase spectrum would obtain in this way, superior results.For inversion, the tests indicated Bayesian as the most efficient once it converged to the real model in all tests, even in the most complex situation with wavelet estimated and noise, though artifacts appeared in the inverted data. Anyway this method produces good estimates of the impedance model, delimiting all the interfaces and solving the uncertainties related with both the noise and an estimated wavelet. Summarizing, through the tests carried out, the inversions methods performed well under a low noise level situation (which can be accomplished through robust pre-conditioning techniques) and showed uncertainty in the wavelet estimation can affect drastically inversion results. Moreover, even though no well information was input to the experiments, statistical wavelets provided good results. Finally It can also be inferred that by using a consistent low frequency model these wavelets are valid though a comparison between them and deterministic wavelets in the seismic inversion context should be performed to confirm this.

\section{ACKNOWLEDGEMENTS}

The authors thank Petrobras for the financial support to the infrastructure of the GISIS (Group of Seismic Inversion and Imaging) Research Group. We are grateful to the members of the Group for the comments and discussions. We also thankful to the Department of Geology and Geophysics of Universidade Federal Fluminense for allowing this work to be completed.

\section{REFERENCES}

[1]. B.H. Russell, Introduction to seismic inversion methods (Society of Exploration Geophysicists, 1988).

[2]. D.A. Cooke, W.A. Schneider, Generalized linear inversion of reflection seismic data, Geophysics, 48(6), 1983, 665-676.

[3]. T.J. Campbell, F.W.B. Richards, R.L. Silva, G. Wach, L. Eliuk, Interpretation of the Penobscot 3D seismic volume using constrained sparse spike inversion, Sable sub-Basin, offshore Nova Scotia, Marine and Petroleum Geology, 68(Part A), 2015, 73-93.

[4]. J.J. Kormylo, J. Mendel, Maximum likelihood detection and estimation of bernoulli-gaussian processes, Information Theory, IEEE Transactions on, 28(3), 1982, 482-488.

[5]. C. Chi, J. Goutsias, J. Mendel, A fast maximum-likelihood estimation and detection algorithm for bernoulli-gaussian processes, Acoustics, Speech, and Signal Processing , IEEE International Conference on ICASSP'85, 10, 1985, 12971300.

[6]. B. Ursin, O. Holberg, Maximum-likelihood estimation of seismic impulse responses, Geophysical Prospecting, 33(2), 1985, 233251.

[7]. D. Oldenburg, T. Scheuer, S. Levy, Recovery of the acoustic impedance from reflection seismograms, Geophysics, 48(10), 1983, 1318-1337.

[8]. M.D. Sacchi, Reweighting strategies in seismic deconvolution, Geophysical Journal International, 129(3), 1997, 651656.

[9]. Y. Wang, Seismic impedance inversion using 11-norm regularization and gradient descent methods, Journal of Inverse and Ill-posed Problems, 18(7), 2011, 823-838.

[10]. T.J. Ulrych, M.D. Sacchi, Informationbased inversion and processing with applications, 36 (Elsevier, 2005).

[11]. O. Osman, E. Robinson, Seismic source signature estimation and measurement, Geophysics Reprint Series (Society of Exploration Geophysicists, 1996).

[12]. E.A. Robinson, Predictive decomposition of time series with applications to seismic exploration, $\mathrm{PhD}$ thesis, Massachusetts Institute of Technology, Cambridge, MA, 1954.

[13]. T. Ulrych, Application of homomorphicdeconvolution to seismology, Geophysics, 36(4), 1971, 650-660.

[14]. A.V. Oppenheim, Superposition in a class of nonlinear systems, Technical Report, DTIC Document, 1965.

[15]. D.R. Velis, T.J. Ulrych, Simulated annealing wavelet estimation via fourthorder cumulant matching, Geophysics, 61(6), 1996, 1939-1948.

[16]. J. Zheng, S. ping Peng, M. chu Liu, Z. Liang, A novel seismic wavelet estimation method, Journal of Applied Geophysics, 90(0), 2013, 92-92.

[17]. B. Yi, G. Lee, H.J. Kim, H.T. Jou, D. Yoo, B. Ryu, K. Lee, Comparison of wavelet estimation methods, Geosciences Journal, 17(1), 2013, 55-63.

[18]. Z. Wang, B. Zhang, J. Gao, The residual phase estimation of a seismic wavelet using 
a rényi divergence-based criterion, Journal of Applied Geophysics, 106(0), 2014, 96105 .

[19]. S.A.M. Oliveira, W.M. Lupinacci, L1 norm inversion method for deconvolution in attenuating media, Geophysical Prospecting, 61(4), 2013, 771-777.

[20]. S. Levy, D. Oldenburg, Automatic phase correction of common-midpoint stacked data, Geophysics, 52(1), 1987, 51-59.

[21]. O. Yilmaz, Seismic data analysis: processing, inversion and interpretation of seismic data (Society of Exploration Geophysicists, 2nd edition, 2001).

[22]. E.A. Robinson, S. Treitel, Geophysical signal analysis (Society of Exploration Geophysicists, 2000).

[23]. L.R. Lines, T.J. Ulrych, The old and the new in seismic deconvolution and wavelet estimation, Geophysical Prospecting, 25(3), 1977, 512-540.

[24]. N. Wiener, Extrapolation, interpolation, and smoothing of stationary time series: with engineering applications (The MIT Press, 1966).
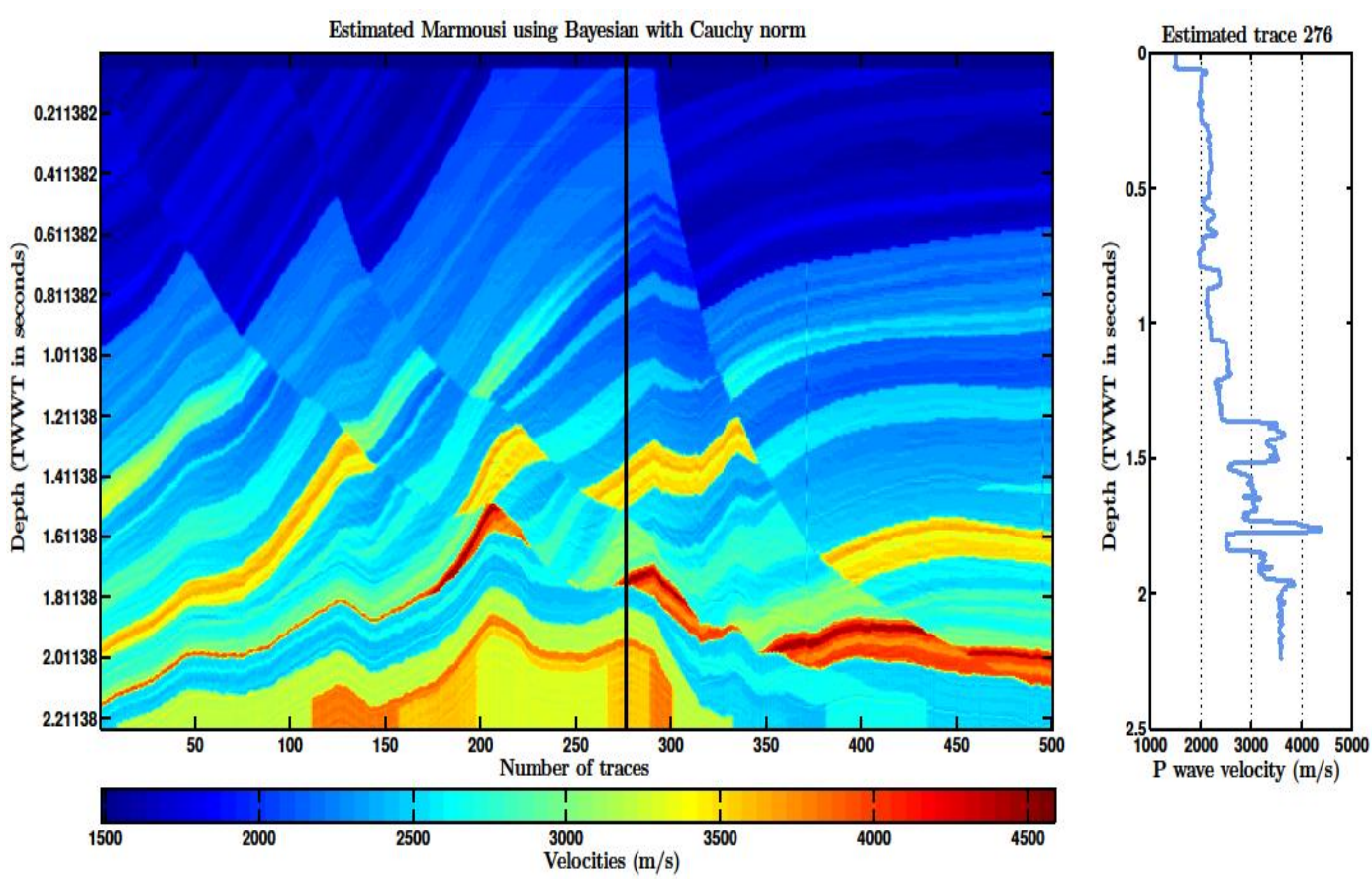

Fig.21 -Marmousi estimated with Bayesian inversion for full seismogram and 1000 iterations per trace.
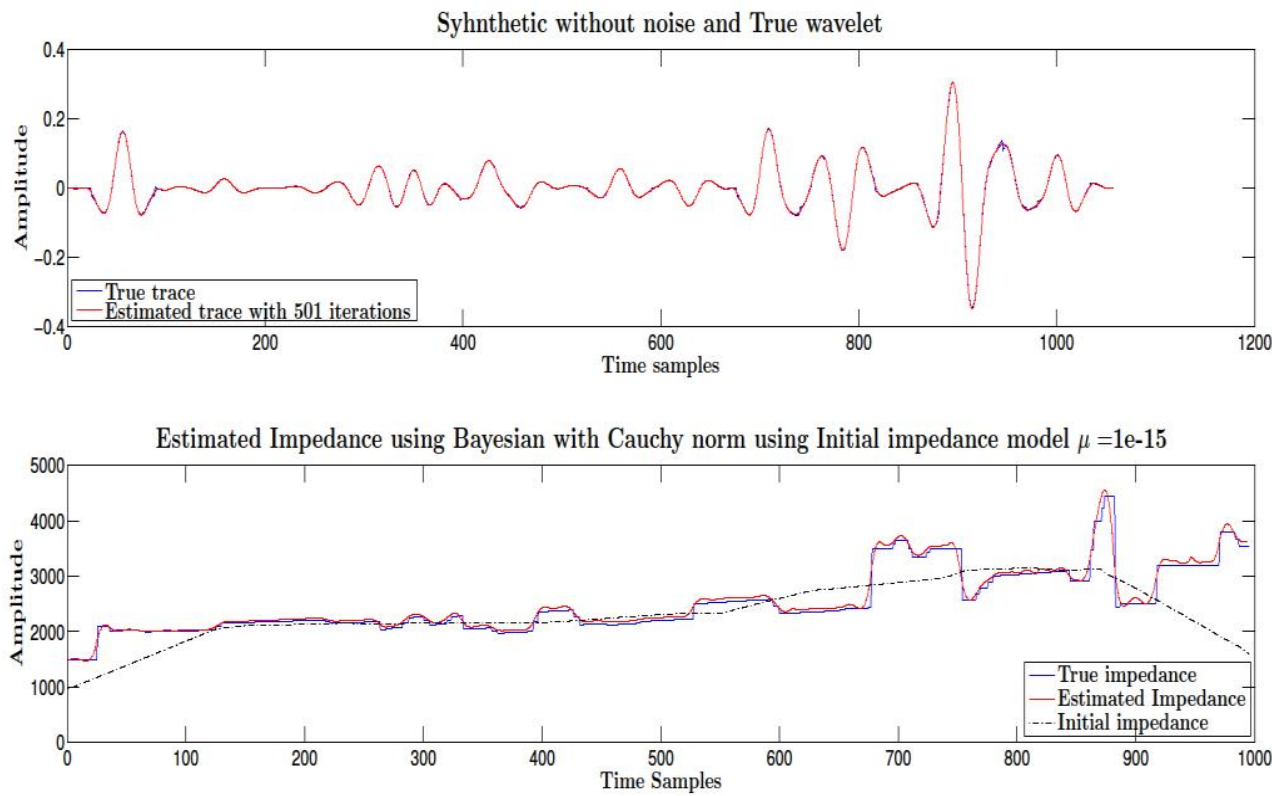
Fig.22 -Marmousi estimated with Bayesian inversion. Trace 276.

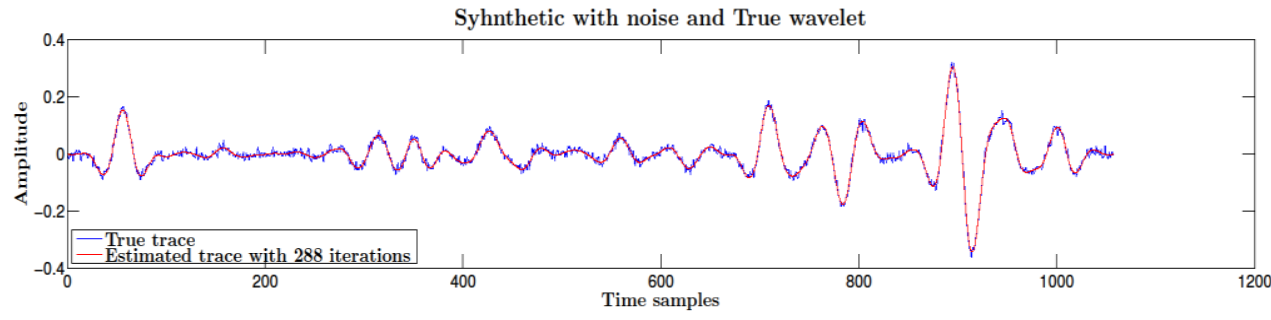

Estimated Impedance using Bayesian with Cauchy norm using Initial impedance model and noise addition $\sigma=10^{-3} \mu=3$

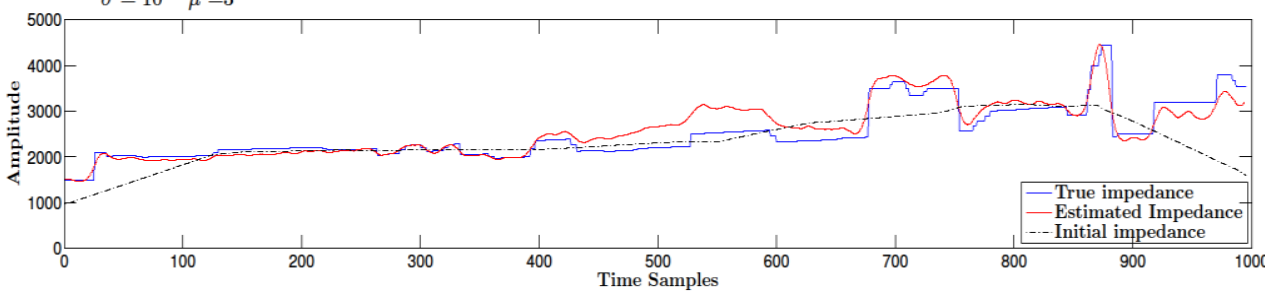

Fig.23 - Marmousi estimated with Bayesian inversion and noise $\sigma=10^{-3}$.

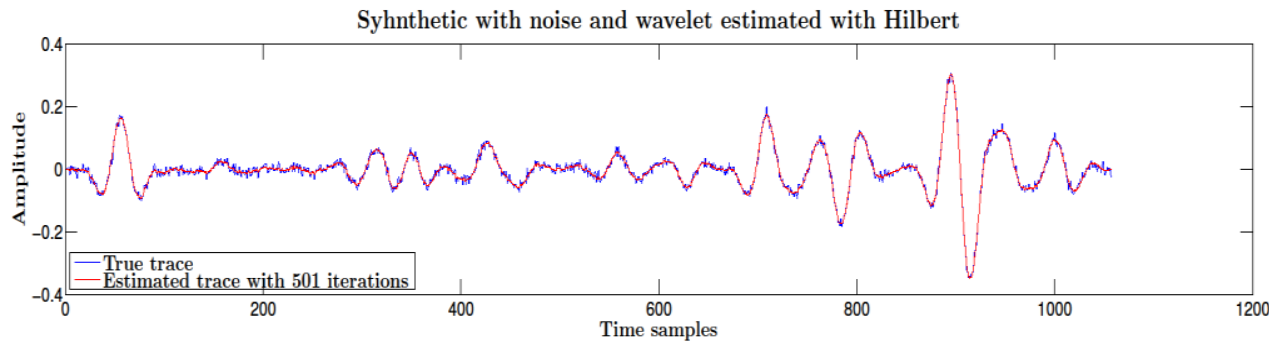

Estimated Impedance using Bayesian with Cauchy norm using Initial impedance model and noise addition $\left(\sigma=10^{-3}\right), \mu=8.9525 \mathrm{e}-05$

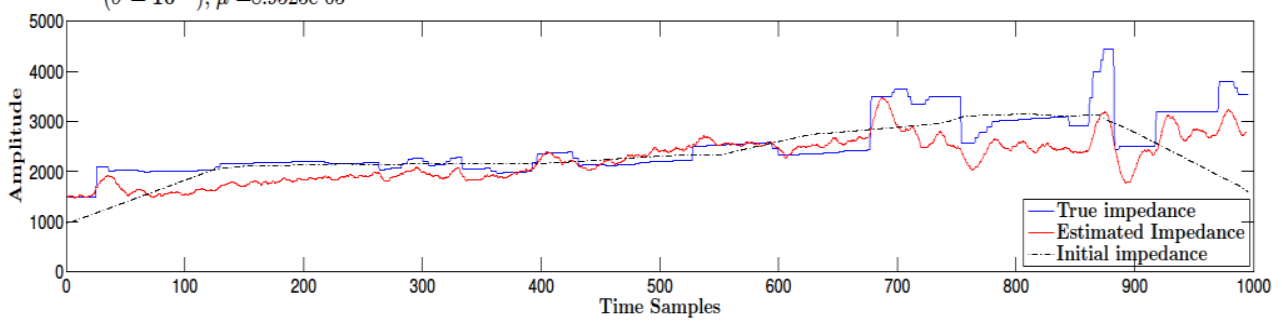

Fig.24 - Marmousi estimated with Bayesian inversion using Hilbert transform method for wavelet estimation and noise $\left(\sigma=10^{-3}\right)$.

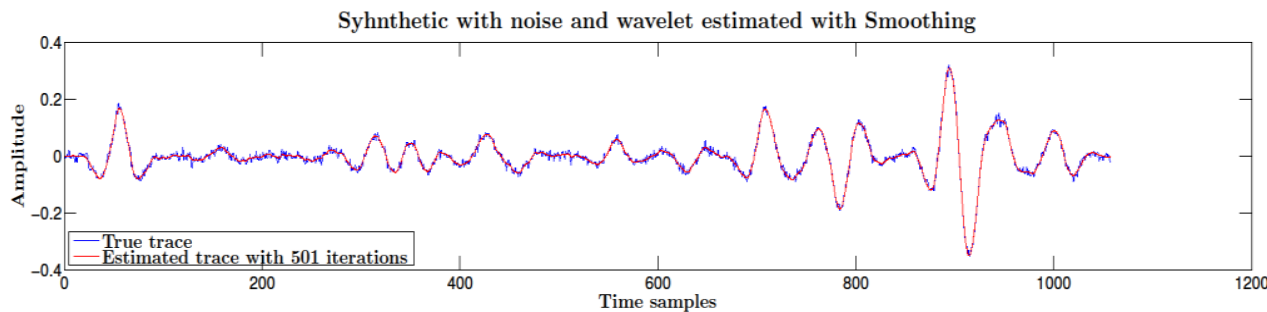

Estimated Impedance using Bayesian with Cauchy norm using Initial impedance model and noise addition $\left(\sigma=10^{-3}\right), \mu=0.00051$

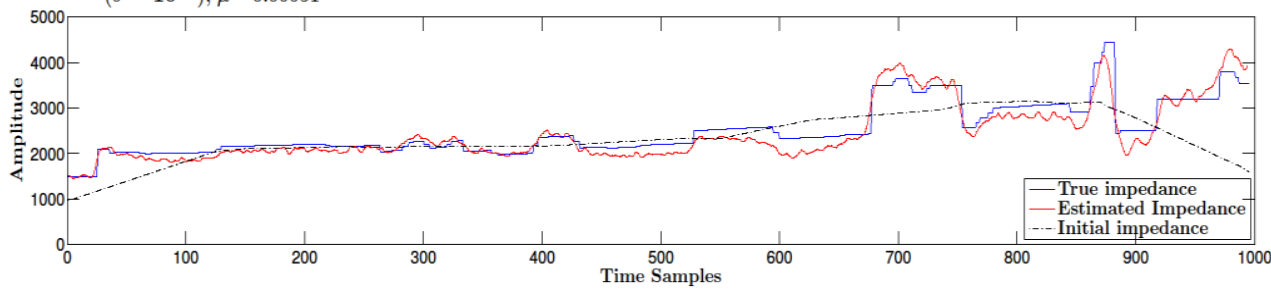


Fig.25 - Marmousi estimated with Bayesian inversion using Smooth spectra method for wavelet estimation and noise $\left(\sigma=10^{-3}\right)$.

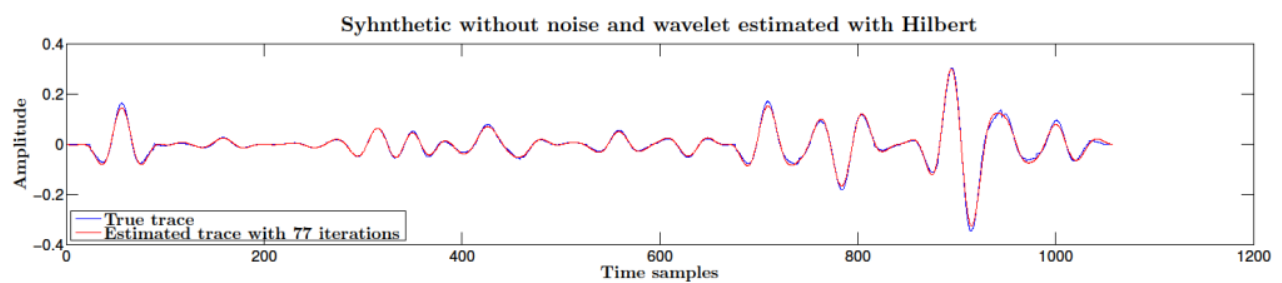

Estimated Impedance using Bayesian with Cauchy norm using Initial impedance model and wavelet estimated with Hilbert. $\mu=2.5 \mathrm{e}-05$

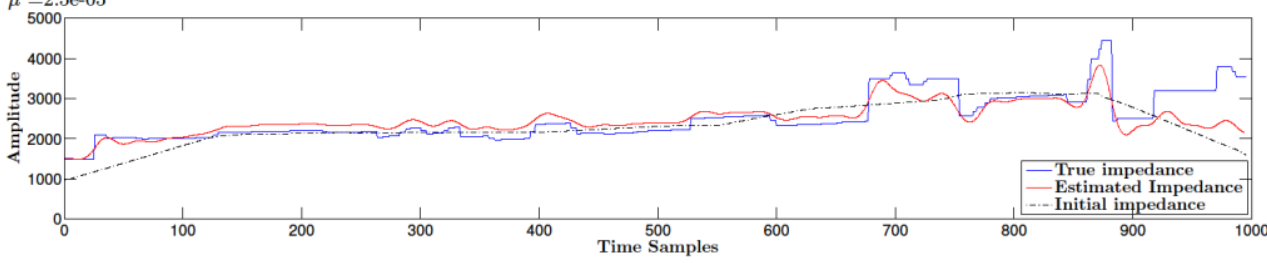

Fig.26 - Marmousi estimated with Bayesian inversion using Hilbert transform method for wavelet estimation without noise.

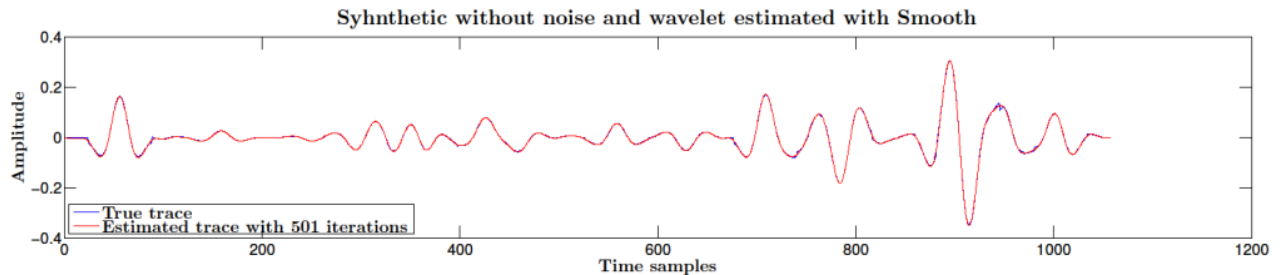

Estimated Impedance using Bayesian with Cauchy norm using Initial impedance model and wavelet estimated with Smooth. $\mu=6 \mathrm{e}-06$

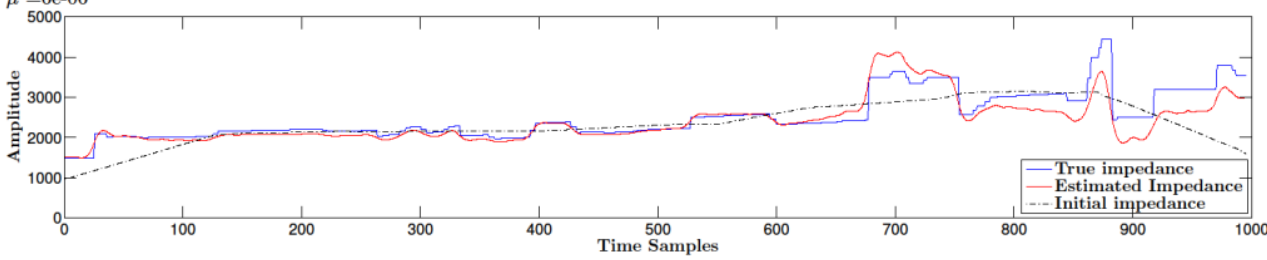

Fig.27 -Marmousi estimated with Bayesian inversion using Smooth spectra method for wavelet estimation and without noise. 